\title{
Magnetically induced Rayleigh-Taylor instability under rotation: Comparison of experimental and theoretical results
}

\author{
M. M. Scase, ${ }^{1}$ K. A. Baldwin, ${ }^{2,3}$ and R. J. A. Hill ${ }^{2, *}$ \\ ${ }^{1}$ School of Mathematical Sciences, University of Nottingham, Nottingham NG7 2RD, United Kingdom \\ ${ }^{2}$ School of Physics and Astronomy, University of Nottingham, Nottingham NG7 2RD, United Kingdom \\ ${ }^{3}$ School of Science and Technology, Nottingham Trent University, Nottingham NG11 8NS, United Kingdom
}

(Received 27 September 2019; revised 20 December 2019; accepted 31 March 2020; published 2 October 2020)

\begin{abstract}
Our theoretical work has shown that rotating a Rayleigh-Taylor-unstable two-layer stratification about a vertical axis slows the development of the instability under gravity and can stabilize axisymmetric modes indefinitely. Here we compare theoretical predictions directly with our experiments on a rotating two-layer system which is made unstable by magnetic forces applied using a superconducting magnet.
\end{abstract}

DOI: 10.1103/PhysRevE.102.043101

\section{INTRODUCTION}

The Rayleigh-Taylor instability (RTI) occurs at the interface between two layers of fluid of different density subjected to gravity, when the denser layer lies over the lighter one. It may also occur under acceleration of the system, when the less dense of the two layers is accelerated in the direction of the more dense one. A layer of salt water lying over a layer of fresh water provides a simple example from a long list of natural phenomena and industrial processes in which this instability occurs [1,2]. Unless the interface is stabilized by some means, for example, by interfacial tension, small deviations of the interface from equilibrium grow rapidly into larger structures, sometimes referred to as "bubbles" or "spikes," as the fluids interpenetrate. The ability to impose some form of constraint or control over the RTI, beyond setting the initial density difference or altering the interfacial tension, would be beneficial in a number of situations where it occurs. In particular, the difficulties arising from the RTI in efforts to generate power from inertial confinement fusion [1] have motivated previous researchers to seek to understand the influence of rotation on the RTI (e.g., Refs. $[3,4]$ ). The Coriolis force acting on fluids under rotation is known to have a stabilizing effect on otherwise unstable fluid flows. Theoretical studies of centrifuged fluids showed that rotation about an axis perpendicular to the direction of acceleration could inhibit RTI [3,4].

Here we discuss our experimental and theoretical studies of RTI in which the rotation axis is parallel to the direction of gravity. Previously, we studied experimentally the onset of the

*richard.hill@ nottingham.ac.uk

Published by the American Physical Society under the terms of the Creative Commons Attribution 4.0 International license. Further distribution of this work must maintain attribution to the author(s) and the published article's title, journal citation, and DOI.
RTI in a two-layer system under rigid-body rotation around a vertical axis [5]. We used magnetic forces to destabilize the system in order to avoid technical difficulties in using standard experimental techniques, such as barrier removal [6] or linear acceleration [7], to initiate RTI in a rotating system. A weakly paramagnetic, light liquid upper layer was floated on a denser diamagnetic liquid layer. The liquids used were miscible so that there was no interfacial tension between the two liquid layers. A superconducting solenoid magnet situated beneath the liquids exerted an attractive magnetic force on the paramagnetic layer and a repulsive magnetic force on the diamagnetic layer. A schematic diagram of the set-up is shown in Fig. 1. The tank containing the liquids was lowered toward the solenoid until the magnetic forces increased sufficiently in magnitude for the system to become Rayleigh-Taylor unstable as shown in the photographs in Fig. 2. These images show the development of the instability, with "bubbles" of the paramagnetic layer (dyed fluorescent green) intruding into the diamagnetic layer below it (colorless). In the upper row of images, the container is not rotating; in the lower row of images, the cylindrical container is rotating at approximately 24 revolutions per minute, around its vertical axis. Compared to the nonrotating system, the length scale of instability in the rotating system is reduced.

In Ref. [8] we showed theoretically that rotation around a vertical axis may retard the development of the RTI under gravity (without magnetic forces) and found that axisymmetric modes may be stabilized indefinitely.

Here we compare our experimental data and theoretical results. In Sec. II we present the model. First, we consider the effect of the magnetic forces on the dispersion relation of interfacial oscillations in a nonrotating system, arriving at the definition of an effective gravity. Second, we introduce the effective gravity into the theoretical results given in Ref. [8] and obtain a critical angular velocity for the stabilization of axisymmetric modes in the experimental system. In Sec. III we present the experimental set-up and summarize our experimental results. In Sec. IV, we present an analysis of 


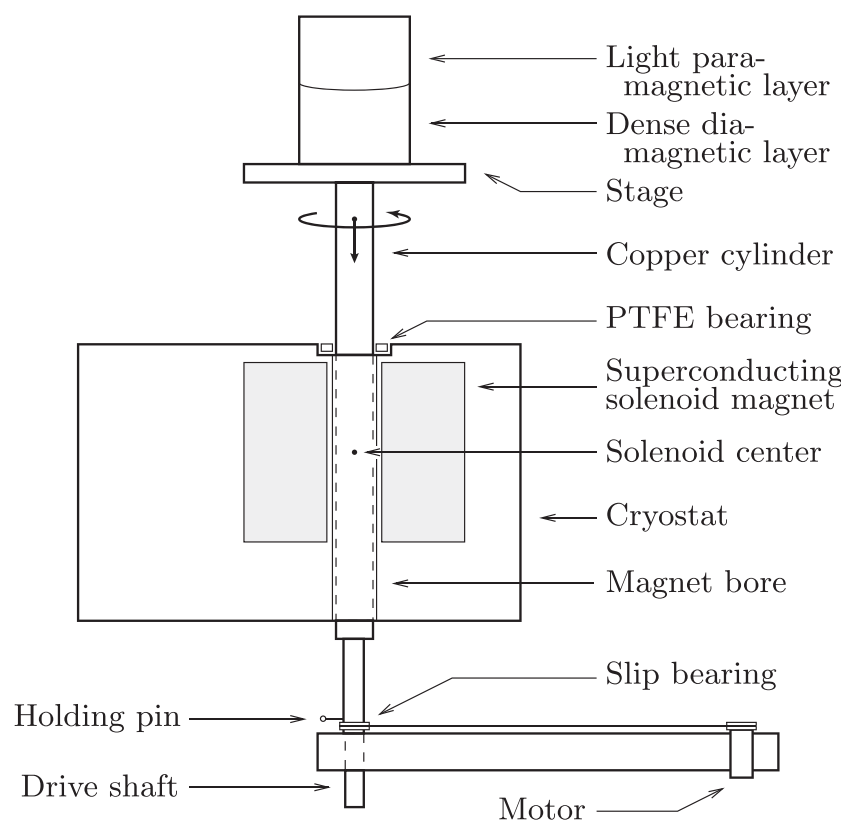

PTFE buffer

FIG. 1. Experimental arrangement (not to scale). A gravitationally stable two-layer stratification was spun-up above the magnet by a motor. Once a holding pin was removed, the system descended slowly $\left(\sim 10 \mathrm{~mm} \mathrm{~s}^{-1}\right)$ into the magnetic field. Slip rings allowed the system to descend while maintaining a constant angular velocity.

the experimental results obtained in Ref. [5] in the context of our theoretical predictions, making a direct comparison between the calculated and experimentally observed angular velocity required to stabilize the longest wavelength mode of instability.

\section{MODEL}

\section{A. Dispersion relation in a gradient magnetic field}

In both experiment and in the theoretical model, we consider a system comprising two miscible liquids (i.e., with no interfacial tension), a layer " 1 " of density $\rho_{1}$ lying over a layer " 2 " of density $\rho_{2}$, as shown schematically in Fig. 3. In the absence of magnetic forces, the dispersion relation for an interfacial perturbation of the form $\exp [i(\kappa x+\omega t)]$ (i.e., with wave number $\kappa$ ) is [9]

$$
\omega^{2}=g A \kappa \tanh (\kappa h)
$$

for layers having equal thickness $h$. Here $g$ is the standard acceleration due to gravity $\left(9.8 \mathrm{~ms}^{-2}\right)$ and $A$ is the Atwood number, which we define as

$$
A=\frac{\rho_{2}-\rho_{1}}{\rho_{2}+\rho_{1}} .
$$

If $A>0$, then the interface supports oscillations, with gravity providing the restoring force. If $A<0$, then interfacial perturbations grow rather than oscillate, i.e., the system is Rayleigh-Taylor unstable.

We now consider the effect on the dispersion relation of the magnetic forces applied in our experiments. Layers 1 and
2 have volume magnetic susceptibility $\chi_{1}$ and $\chi_{2}$, respectively (Fig. 3). We suppose that $|\chi| \ll 1$, since $|\chi|$ is of order $10^{-5}$ in both layers in our experiments. In this case, the magnetic force $\boldsymbol{f}_{m}$ on a volume $d V$ of fluid subjected to a magnetic field $\mathbf{B}$ of strength $B=|\mathbf{B}|$ is, to a good approximation [10,11],

$$
f_{m} d V=\chi \frac{\nabla B^{2}}{2 \mu_{0}} d V .
$$

Note that the direction of the force is given by the gradient of the strength of the magnetic field, not by the direction of the vector field B. Such magnetic body forces give rise to phenomena such as the "Moses effect" [12] and magnetically enhanced buoyancy [13-15] (magneto-Archimedes effect) in fluids and have also been exploited to levitate fluids in weightlessness (see, for example, Refs. [10,13,16-19]). In our experiments on the RTI, the magnetic field was generated by a superconducting solenoid with a vertical axis. The solenoid produces a cylindrically symmetric magnetic field of strength $B(r, z)$, where $r$ and $z$ are radial and vertical cylindrical coordinates, respectively. The experiments were performed in a region above the center of the solenoid coil where $B$ decreases with increasing height $z$ (i.e., $\partial B / \partial z<0$ ) but varies relatively weakly with radius (i.e., $|\partial B / \partial z| \gg|\partial B / \partial r|)$. We thus make the approximation that, in this region,

$$
\left.\boldsymbol{f}_{m}(r, z) d V \approx \frac{\chi}{2 \mu_{0}} \frac{\partial B^{2}}{\partial z}\right|_{r=0} \hat{z} d V,
$$

i.e., neglecting the radial variation of $B^{2}$. We further assume that $\frac{\partial B^{2}}{\partial z}$ can be taken to be a constant to a good approximation in the interfacial region. In this case we approximate the Euler equation in the interfacial region by

$$
\rho_{j} \frac{D \boldsymbol{u}_{j}}{D t} \approx-\nabla p_{j}+\frac{\chi_{j}}{2 \mu_{0}} \frac{\partial B^{2}}{\partial z} \hat{z}-\rho_{j} g \hat{z}
$$

for the two layers $j=1,2$, where the derivative $\partial B^{2} / \partial z$ is evaluated at the equilibrium position of the interface at the origin. We assume that the liquids are incompressible so that $\boldsymbol{\nabla} \cdot \boldsymbol{u}_{j}=0$. Imposing pressure continuity across the interface, we obtain the dispersion relation for equal layer depths $h=$ $h_{1}=h_{2}$ (see Appendix)

$$
\begin{aligned}
\omega^{2} & =g A \kappa \tanh (\kappa h)\left[1-\frac{1}{2 g \mu_{0}}\left(\frac{\chi_{2}-\chi_{1}}{\rho_{2}-\rho_{1}}\right) \frac{\partial B^{2}}{\partial z}\right] \\
& =\Gamma A \kappa \tanh (\kappa h),
\end{aligned}
$$

defining the effective gravity acting at the interface, $\Gamma$, as

$$
\Gamma=g\left[1-\frac{1}{2 g \mu_{0}}\left(\frac{\chi_{2}-\chi_{1}}{\rho_{2}-\rho_{1}}\right) \frac{\partial B^{2}}{\partial z}\right] .
$$

Again, the derivative is to be evaluated at the equilibrium position of the interface at the origin. The sign of $\Gamma$ indicates the direction of the effective gravity; where $\Gamma<0$, the effective gravitational force at the interface points in the opposite direction to that of standard gravity, i.e., vertically upwards.

In (1) $g$ is the magnitude of the gravitational acceleration (i.e., unsigned) and stability is indicated by the sign of the Atwood number. We now write (6) in a corresponding form by 


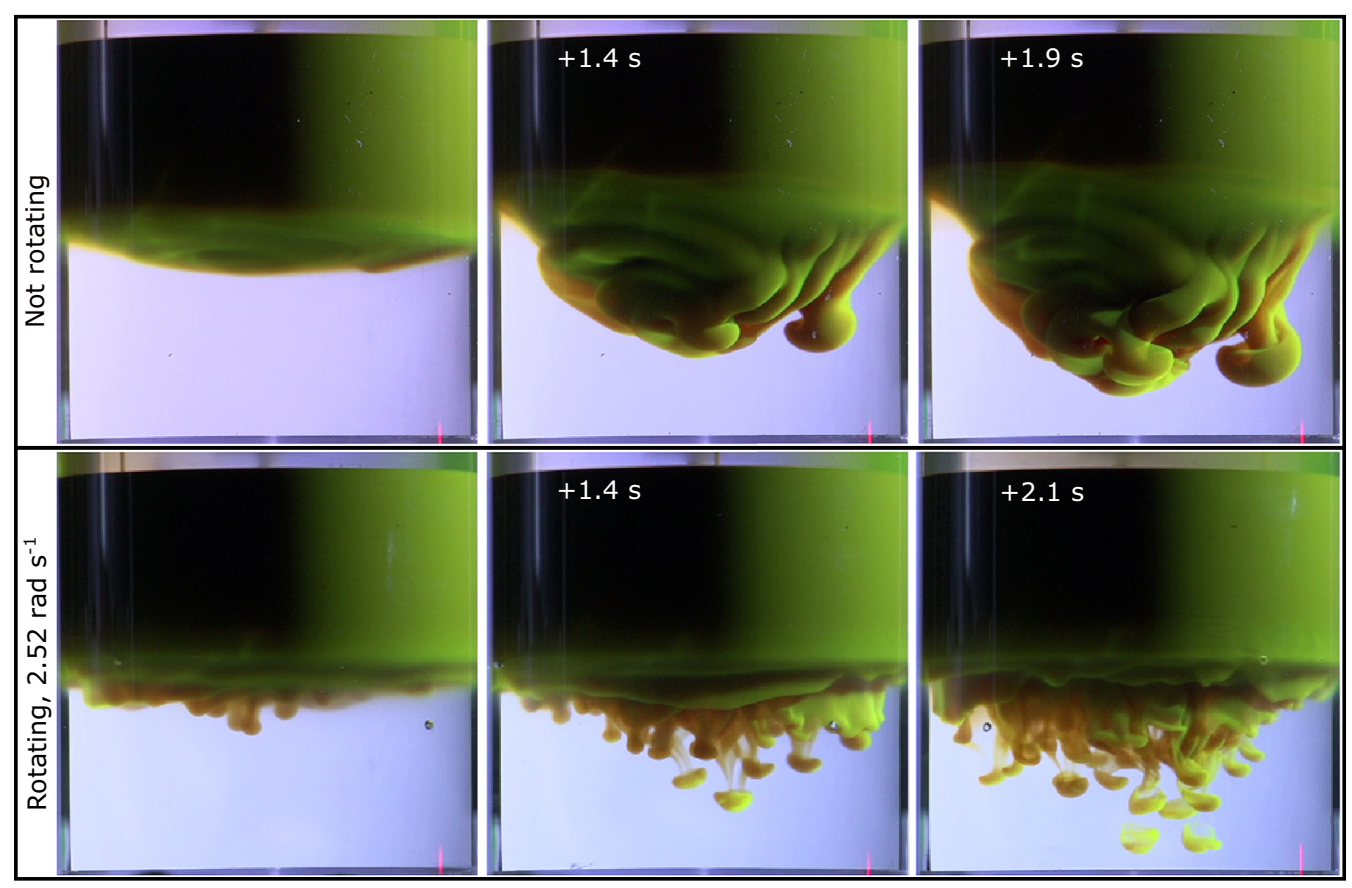

FIG. 2. Rayleigh-Taylor instability developing at the interface between a layer of $0.06 \mathrm{M}$ manganese chloride solution (dyed fluorescent green) lying above a more dense $0.4 \mathrm{M}$ sodium chloride solution (colorless) in a 90-mm diameter cylindrical tank. The instability is caused by the magnetic attraction of the weakly paramagnetic upper layer to a superconducting magnet situated beneath the tank (not visible in these images) and by the magnetic repulsion of the colorless diamagnetic layer from the magnet. In the upper row of images, the cylindrical container is not rotating; in the lower row, the container is rotating at $2.52 \mathrm{rad} \mathrm{s}^{-1}$ about its vertical axis. The images illustrate how rotation restricts the size of the structures that form at the interface.

defining a modified Atwood number $A^{\prime}$ such that $|\Gamma| A^{\prime}=\Gamma A$. Using this definition of the Atwood number, Eq. (6) becomes

$$
\omega^{2}=|\Gamma| A^{\prime} \kappa \tanh (\kappa h),
$$

which is the analog of (1) in the gradient magnetic field; i.e., the interface is stable for $A^{\prime}>0$ and unstable for $A^{\prime}<0$ [20].

\section{B. Dispersion relation under rotation}

In Ref. [8] we modelled a system consisting of two layers of equal volume, depth $h$, contained in a cylindrical tank of radius $a$ and height $2 h$, rotating about its axis with angular velocity $\Omega$. In the initial state, the liquid layers were assumed to be rotating as rigid bodies at the same angular velocity as the tank. The two-layer system was subjected to standard gravity but not magnetic forces. The stability of the model system

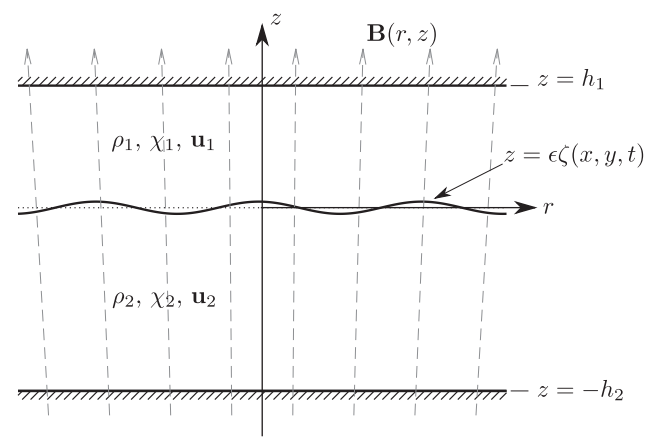

FIG. 3. Schematic of the flow domain. was analyzed as a function of angular velocity and Atwood number. We considered perturbations to the interface of the form $z=z_{0}(r)+\epsilon \zeta(r, \theta, t)$ with normal mode solutions of the form $\zeta=\hat{\zeta}(r) \exp \{i(\omega t+m \theta)\}$.

\section{Axisymmetric modes $(m=0)$}

We first focus on the axisymmetric modes, $m=0$, which we predicted in Ref. [8] could be stabilized by rotation. The axisymmetric modes have a factor

$$
\hat{\zeta}_{n}(r) \propto \mathcal{J}_{0}\left(\frac{k(n) r}{a}\right),
$$

where $\mathcal{J}_{0}$ is a Bessel function of the first kind. The radial impermeability boundary condition at $r=a$ defines the possible modes of solution $k(n)$ : For $m=0$, the $k(n)$ are those that satisfy $\mathcal{J}_{1}(k(n))=0$ for $n=1,2,3, \ldots$. Proceeding with the analysis given in Ref. [8], but with the addition of the magnetic body force (4) to the (rotating) Euler equation, leads to the same conclusions given in Ref. [8], except that $|\Gamma|$ is substituted for $g$ in the expressions, where $\Gamma$ is as defined in (7). The dispersion relation for the axisymmetric modes in a nonrotating tank is (substituting $|\Gamma|$ for $g$ and $A^{\prime}$ for $A$ in Ref. [8])

$$
\omega^{2}=|\Gamma| A^{\prime} \frac{k(n)}{a} \tanh [k(n) \delta],
$$

where $\delta=h / a$. The ratio $k(n) / a$ may be regarded as the radial wave number associated with the $n$th mode. We obtain the dispersion relation of axisymmetric modes for small rotation 
rates, $\Omega^{2} a /|\Gamma| \ll 1$, by series expansion [8]:

$$
\begin{aligned}
\omega^{2} \sim & \frac{|\Gamma|}{a} A^{\prime} k \tanh (k \delta)+2 \Omega^{2}[1+2 k \delta \operatorname{csch}(2 k \delta) \\
& \left.-\frac{1}{24} k^{2} A^{\prime 2} \operatorname{sech}^{2}(k \delta)\right]+\frac{|\Gamma|}{a} O\left(\left[\frac{\Omega^{2} a}{|\Gamma|}\right]^{2}\right),
\end{aligned}
$$

where $k=k(n)$. The form of (11) suggests that rotating the system may suppress the growth of the RTI in an intrinsically unstable system $\left(A^{\prime}<0\right)$. Indeed, we found a general expression (i.e., not asymptotic) [8] for the critical angular velocity $\Omega_{c}$ for axisymmetric modes, such that the mode $k(n)$ is stabilized for $\Omega>\Omega_{c}$. In the gradient magnetic field, the expression for the critical angular velocity is

$$
\frac{\Omega_{c}^{2} a}{|\Gamma|}=\frac{6 \delta}{A^{\prime}}\left(1-\frac{k^{2}}{48}\right)^{-1}\left[\left\{1-\frac{k^{2} A^{\prime 2}}{12}\left(1-\frac{k^{2}}{48}\right)\right\}^{1 / 2}-1\right]
$$

where $k=k(n)$.

\section{Nonaxisymmetric modes $(\boldsymbol{m} \neq \mathbf{0})$}

We now consider the nonaxisymmetric modes. Similarly to (9) the nonaxisymmetric modes have a factor

$$
\hat{\zeta}_{n}(r) \propto \mathcal{J}_{m}\left(\frac{k(n) r}{a}\right) .
$$

The radial impermeability condition forces the radial wave number, $k$, to satisfy

$$
k \mathcal{J}_{m+1}(k)=m\left(1+\frac{2 \Omega}{\omega}\right) \mathcal{J}_{m}(k)
$$

In the axisymmetric case, $m=0$, (14) simplifies and $k$ is real and independent of $\omega$. However, in the general nonaxisymmetric case we see that $k$ is a function of $\omega$ and may be complex. We therefore consider simultaneous asymptotic expansions in both the wave number $k$ and the growth rate $\omega$. Following the method of Scase, Baldwin, and Hill [8] we expand the wave number, $k$, and growth rate, $\omega$, in terms of a nondimensional rotation rate $\alpha=\Omega^{2} a /|\Gamma|$ as

$$
\begin{gathered}
k \sim k_{0}+k_{1} \alpha^{1 / 2}+k_{2} \alpha+O\left(\alpha^{3 / 2}\right), \\
\omega \sim \omega_{0}+\omega_{1} \alpha^{1 / 2}+\omega_{2} \alpha+O\left(\alpha^{3 / 2}\right),
\end{gathered}
$$

where the nonrotating growth rate, $\omega_{0}$, and the real wave number, $k_{0}$, are given by

$$
\begin{gathered}
\left(\frac{a}{|\Gamma|}\right) \omega_{0}^{2}=A^{\prime} k_{0} \tanh \left(k_{0} \delta\right), \\
k_{0} \mathcal{J}_{m+1}\left(k_{0}\right)=m \mathcal{J}_{m}\left(k_{0}\right) .
\end{gathered}
$$

The first-order corrections to both the wave number and the growth rate are given respectively by

$$
\begin{gathered}
\left(\frac{a}{|\Gamma|}\right)^{1 / 2} \omega_{0} k_{1}=\frac{2 k_{0} m}{k_{0}^{2}-m^{2}}, \\
\left(\frac{a}{|\Gamma|}\right)^{1 / 2} \omega_{1}=\frac{m}{k_{0}^{2}-m^{2}}\left[1+2 k_{0} \delta \operatorname{csch}\left(2 k_{0} \delta\right)\right] .
\end{gathered}
$$

The preceding equation, (20), shows that the first-order correction to $\omega$ is real and hence only affects the precession of the mode, not the growth rate. The next-order correction to $\omega$ does affect the growth rate and is given by

$$
\begin{aligned}
& \left(\frac{a}{|\Gamma|}\right) \omega_{0} \omega_{2} \\
& =1-\frac{m^{2}}{2\left(k_{0}^{2}-m^{2}\right)}\left[\frac{5 k_{0}^{2}-m^{2}}{\left(k_{0}^{2}-m^{2}\right)^{2}}+A^{\prime 2}\right] \\
& \quad+2 k_{0} \delta\left(1-\frac{m^{2}}{\left(k_{0}^{2}-m^{2}\right)^{2}}\left\{\frac{2 k_{0}^{2}}{k_{0}^{2}-m^{2}}\right.\right. \\
& \left.\left.\quad+2 k_{0} \delta\left[\operatorname{coth}\left(2 k_{0} \delta\right)+\frac{1}{2} \operatorname{csch}\left(2 k_{0} \delta\right)\right]\right\}\right) \operatorname{csch}\left(2 k_{0} \delta\right) \\
& \quad-\frac{k_{0}^{2} A^{\prime 2}}{8}\left[1-\frac{4 k_{0}^{2}}{k_{0}^{2}-m^{2}} G\left(m, k_{0}\right)\right] \operatorname{sech}^{2}\left(k_{0} \delta\right)
\end{aligned}
$$

where

$$
G(m, k)=\int_{0}^{1} \frac{\mathcal{J}_{m}(k x)^{2} x^{3}}{\mathcal{J}_{m}(k)^{2}} d x .
$$

The growth rate $\operatorname{Im}(\omega)$ of a few axisymmetric and nonaxisymmetric modes $n<=8$ has been plotted in Fig. 4 , for small values of the nondimensional angular velocity $\alpha$. The general trend shows that increasing $\alpha$ increases $\operatorname{Im}(\omega)$; i.e., increasing angular velocity slows the growth of the instability. For fixed values of $m$, in general, the gradient of $\operatorname{Im}[\omega(\alpha)]$ is steeper for modes with smaller $n$; and for fixed values of $n$, in general, the gradient of $\operatorname{Im}[\omega(\alpha)]$ is steeper for modes with smaller $m$. There are exceptions to this pattern: for $n>1$ the growth rate of $m=0$ modes is approximately equal to the growth rate of $m=2$ modes, and the $n=1, m=1,2,3, \ldots$, modes are less influenced by rotation than some modes with greater $n$. Nevertheless, the general trend for small angular velocity is clear, that rotation tends to suppress modes with larger radial and azimuthal length scale (smaller $n$ and $m$ ) more than for modes with smaller length scales (larger $n, m$ ). Although these plots suggest that the growth of nonaxisymmetric modes may be supressed by rotation at higher angular velocity than we have considered here, it would be a mistake to extrapolate from these results that an unstable nonaxisymmetric mode can be stabilized fully by rotation $\left(\omega^{2}>0\right)$, as was shown for the axisymmetric modes (12).

\section{EXPERIMENTAL STUDY}

For the purposes of comparing our experiments with theory, we first describe briefly the experimental method, including the preparation and initial conditions of the two-fluid system, before discussing the experimental results. 


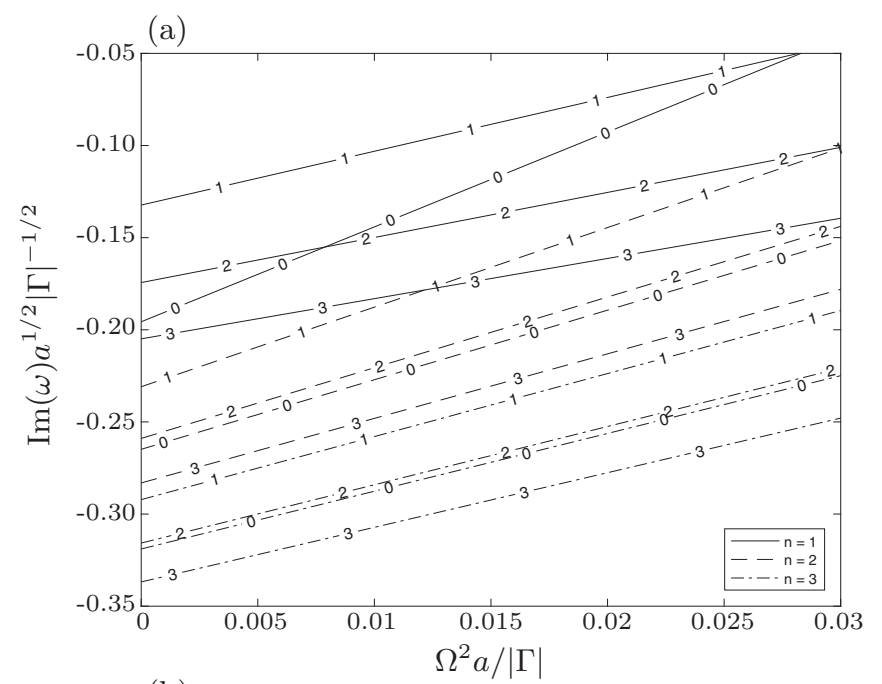

(b)

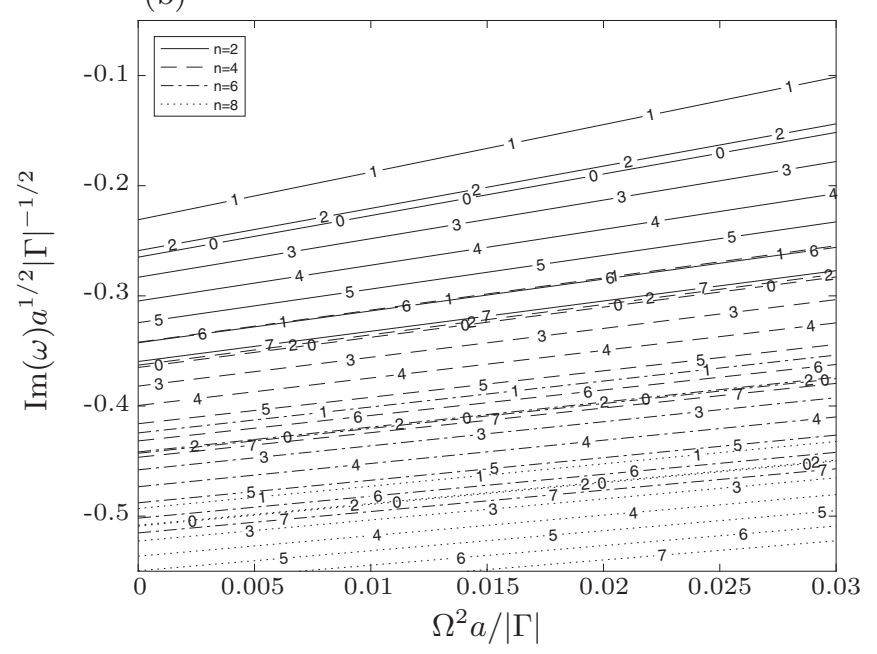

FIG. 4. The growth rate of various axisymmetric and nonaxisymmetric modes of instability. The azimuthal mode of instability $(m)$ is indicated on each solution, the radial wave number $(n)$ is indicated by the legend. For clarity, modes $n<=3$ are shown in panel (a), and modes $n>=2$ ( $n$ even) are shown in panel (b). The parameters chosen for this plot were $A^{\prime}=-0.01, a=1, d=1,|\Gamma|=1$. The effect of the rotation is to decrease the growth rate of the mode, as shown by the positive gradient of each line.

\section{A. Method}

The RTI is known to be sensitive to initial conditions [21]. Care was taken to ensure repeatable initial conditions in these experiments. The experimental system was prepared by pouring a layer of dilute manganese chloride solution (nominally $0.06 \mathrm{M}$ ) onto a layer of higher density sodium chloride solution (nominally $0.4 \mathrm{M}$ ). The upper layer was poured slowly through a floating sponge resulting in a gravitationally stable system of two liquid layers with a well-defined interface between the two. The paramagnetic $\mathrm{Mn}^{2+}$ ions in the upper layer made this layer weakly paramagnetic. We note that, although the liquids move through a strong magnetic field, magnetohydrodynamic effects can be mostly neglected in these experiments due to the relatively low conductivity of the aqueous liquids (conductivity $\sigma \approx 4 \mathrm{Sm}^{-1}$ ); this point is discussed further in Sec. IV.

After the stable stratification was prepared, the system was gradually spun up into rigid body rotation using a motor drive. The process of pouring and spinning up the liquids was performed slowly, over a period of approximately $2 \mathrm{~h}$, to avoid mixing the two miscible liquids. Although we avoided largescale mixing, the interface became slightly diffuse during this time, which was unavoidable. The thickness of the diffusion layer was approximately $2 \mathrm{~mm}$ after $2 \mathrm{~h}$. Since the same procedure was used each time to prepare the system, the width of the diffusion layer was the same in each experimental run. Once rotating at the required angular velocity, the tank was lowered, at $\sim 10 \mathrm{~mm} \mathrm{~s}^{-1}$, into the strong magnetic field generated by a superconducting solenoid magnet (Cryogenic Ltd., London, UK); a schematic of the set-up is shown in Fig. 1. The fall was slowed by magnetic damping resulting from eddy currents flowing in the copper cylinder supporting the tank, as it moved through the magnetic field (Fig. 1). The rate of fall was set precisely by the addition of small weights to the tank before spin-up. The tank descended at a constant speed until reaching a buffer (Fig. 1) that halted its vertical motion but allowed rotation to continue. The tank's vertical motion was stopped in a region of magnetic field in which the effective gravity was inverted, whereupon the system became Rayleigh-Taylor unstable as shown in the photographs in Fig. 2. In all experiments, the depth and concentration of the liquid layers, the strength of the magnetic field applied by the superconducting solenoid, the initial position of the tank above the magnet, the rotation rate, the rate of fall, and the temperature were precisely controlled, ensuring repeatable initial conditions.

\section{B. Structure of the instability}

The images in Fig. 5 show the development of the Rayleigh-Taylor instability for five angular velocities between $1.0 \mathrm{rad} \mathrm{s}^{-1}$ and $13.2 \mathrm{rad} \mathrm{s}^{-1}$. At early times, $t \approx 0.5-2.0 \mathrm{~s}$, after the onset of the instability, a perturbation to the interface can be seen in the images, which exhibits a dominant length scale. At the lowest angular velocity, the structure of the instability is cell-like, as can be seen, for example, in the the images of the system rotating at $\Omega=1.06 \mathrm{rad} \mathrm{s}^{-1}$. At higher angular velocities the cell-like structures are replaced by structures reminiscent of snake-like convection rolls (e.g., Ref. [22]), as seen in the images of the system rotating at $\Omega=$ $3.32 \mathrm{rad} \mathrm{s}^{-1}$ and $\Omega=6.47 \mathrm{rad} \mathrm{s}^{-1}$. The lateral length scale of the instability decreases with increasing angular velocity as is evident if one compares, for example, the structures in the $\Omega=3.32 \mathrm{rad} \mathrm{s}^{-1}$ experiments with those of the $\Omega=$ $8.74 \mathrm{rad} \mathrm{s}^{-1}$ experiments.

A measurement of the dominant length scale of the instability was obtained by analyzing the autocorrelation of the images of the interface, in the region $r<30 \mathrm{~mm}$ [5]. Figure 6 shows that the dominant length scale decreases with increasing angular velocity. The largest change in length scale occurs between 0 and approximately $2 \mathrm{rad} \mathrm{s}^{-1}$. At higher angular velocity, the length scale decreases more slowly, appearing to asymptote to a value of approximately $6 \mathrm{~mm}$. Experiments in which we added glycerol to the solutions showed that the 


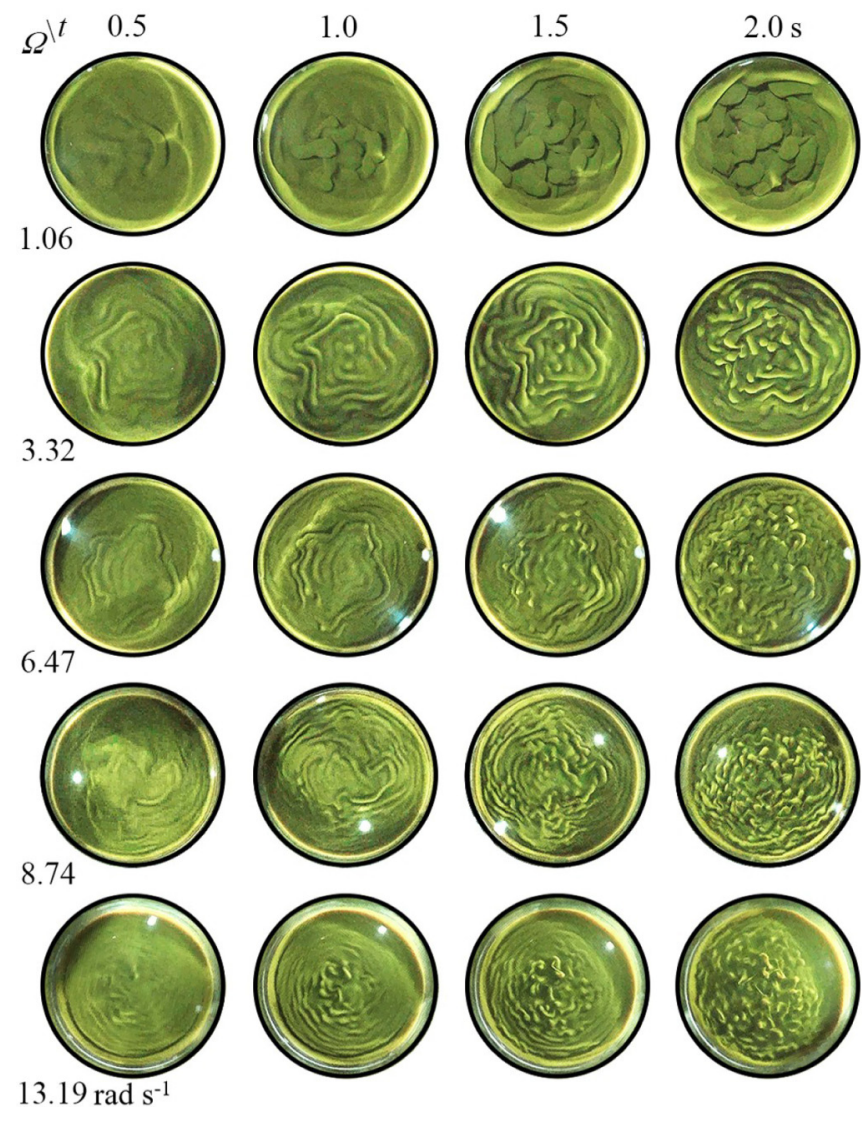

FIG. 5. Images of the interface between the two liquids under rotation, viewed from above. The images show the development of the Rayleigh-Taylor instability with time $t$ after the onset of the instability, increasing from $t=0.5 \mathrm{~s}$ to $2.0 \mathrm{~s}$ left to right, and at different angular velocities, increasing from $\Omega=1.06 \mathrm{rad} \mathrm{s}^{-1}$ to $13.19 \mathrm{rad} \mathrm{s}^{-1}$ top to bottom. The black circles bounding each image highlight the edge of the cylindrical tank (radius $53.5 \mathrm{~mm}$ in these experiments).

asymptotic value of the length scale depends on the viscosity of the liquid layers [5].

\section{Suppression of the instability by rotation}

Figure 7 shows a plot of the vertical location of the interface at $r=0$, relative to its equilibrium position, versus time after the onset of the RTI, for angular velocities in the range $0<\Omega<9.06 \mathrm{rad} \mathrm{s}^{-1}$. In this plot the time when the vertical motion of the tank ceased is at $t=0$. The plot shows a clear trend towards slower growth of the RTI with increasing angular velocity.

We now examine the suppression by rotation of mode $n=1, m=0$, i.e., the axisymmetric mode with the largest radial length scale. The suppression of this mode can be readily observed in Fig. 2. Figure 8 shows the proportional contribution of this mode to the interface profile when the instability has an amplitude of $0.05 h$. The data were obtained by first stretching the experimental images to remove the effects of rotation such that $r \mapsto r, z \mapsto z-z_{0}(r)$ so that the initial approximately parabolic profile was rendered horizontal. The stretched images were contoured yielding a fit to the interface

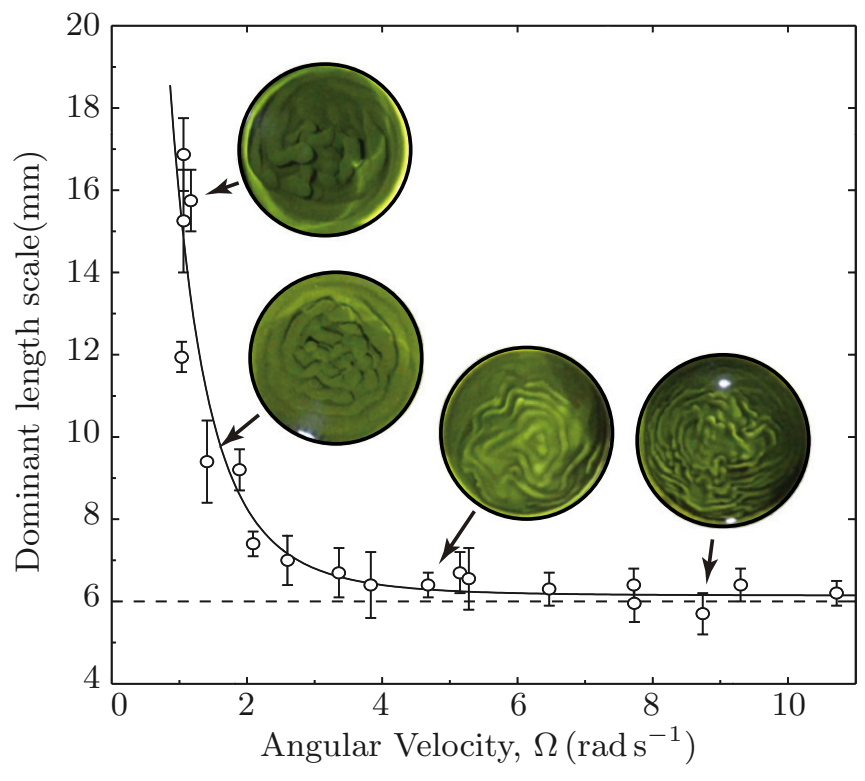

FIG. 6. The dominant scales of perturbation after the onset of instability. Error bars are associated with goodness-of-fit from the spatial autocorrelation algorithm. The solid line is an empirical best fit. Data from Ref. [5].

of the form $z=\zeta(r)$. Coefficients $c_{n}$ were found such that $\zeta(r)=\sum c_{n} \mathcal{J}_{0}\left(k_{n} r / a\right)$. The proportional contribution of the axisymmetric $n=1$ mode was then calculated as $\left|c_{1}\right| / \sum\left|c_{n}\right|$, as plotted in Fig. 8. The data show that the contribution of mode $n=1, m=0$ to the instability decreased sharply above a critical angular velocity $\Omega \approx 1.5 \mathrm{rad} \mathrm{s}^{-1}$ : For $\Omega \lesssim$ $1.5 \mathrm{rad} \mathrm{s}^{-1}$, the proportional contribution of this mode to the instability is significantly greater than for $\Omega \gtrsim 1.5 \mathrm{rad} \mathrm{s}^{-1}$.

In processing the experimental data to obtain the growth rate, we tracked the position of the front of the dyed upper liquid advancing into the undyed lower liquid. This method works well for short times after the onset of the instability, where the deviations from the initial equilibrium interface shape are relatively small. Were we to continue the analysis for longer times after the onset of the instability, until mixing becomes well advanced, a more sophisticated analysis of the mixing width would be required, as discussed recently [23].

\section{DISCUSSION}

We now discuss the results of our experiments in comparison with the predictions of our theoretical model, and consider the relevant assumptions. The dispersion relations derived in the limit of small angular velocities (Sec. II B) suggest that modes of a developing RTI may have their rate of growth inhibited by rotation. Indeed, (12) indicates that axisymmetric modes may be stabilized indefinitely by rotation (as long as the interface does not intersect the base or lid of the container, which provides an upper limit to the rotation speed in the model).

We found that, in experiments, the length scale of the dominant mode decreased with increasing angular velocity (Figs. 5 and 6), and that the overall growth of the instability was slowed by rotation (Fig. 7). These observations are 


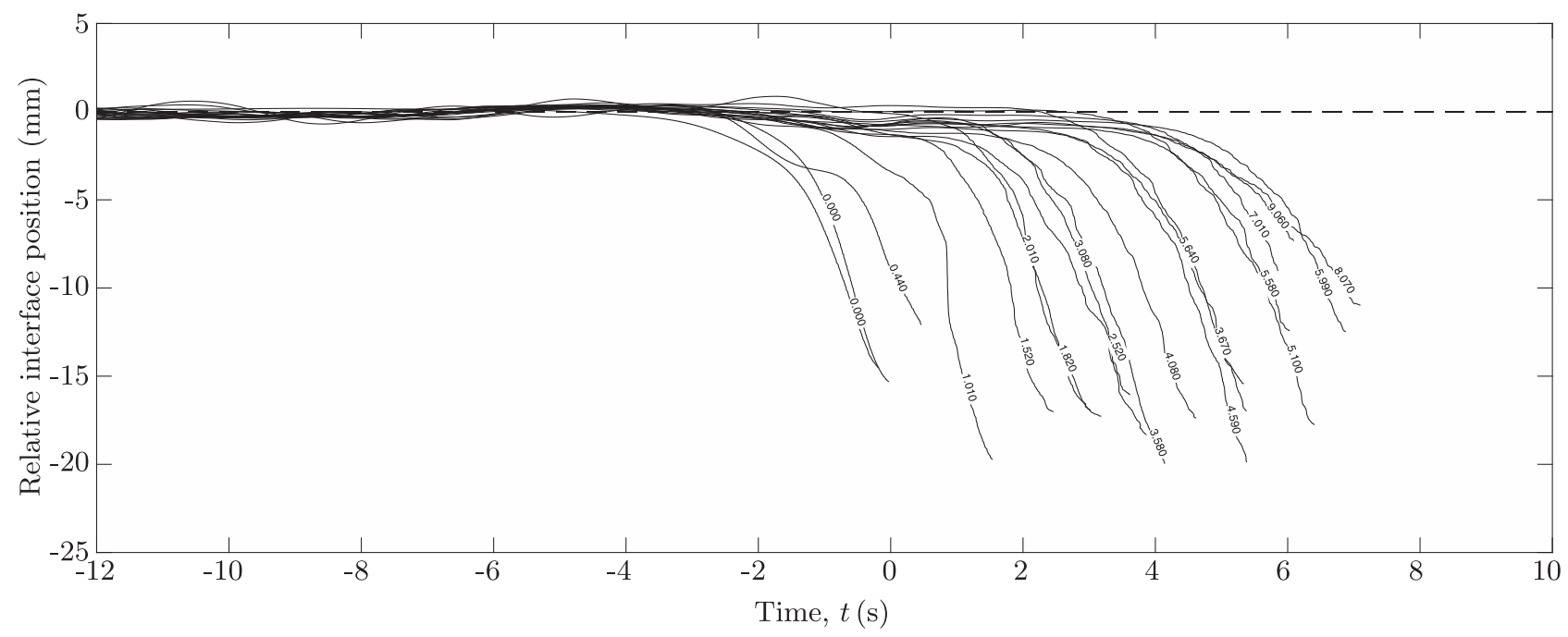

FIG. 7. The position of the interface, relative to the tank, for 20 different angular velocities. Labels on each curve indicate the corresponding angular velocity $\left(\mathrm{rad} \mathrm{s}^{-1}\right)$.

consistent with theory (Sec. II B) indicating that the growth of all modes is typically inhibitted by rotation, and that the growth of modes with larger length scales is inhibitted more by rotation than modes with shorter length scales. Our observations are also consistent with the prediction that some larger wavelength modes are completely stabilized by rotation. The length scale reached a minimum of approximately $6 \mathrm{~mm}$ at an angular velocity of approximately $7 \mathrm{rad} \mathrm{s}^{-1}$, and remained constant for higher angular velocities. Additional experiments showed this minimum length scale to be influenced by the viscosity of the liquids, consistent with previous findings. For example, it has been shown in Ref. [24] that the viscosity of the fluid inhibits the formation of small structures in classical nonrotating RTI: Higher viscosity in the fluid layers leads to larger observed structures in the developing instability. On the basis of our theoretical and experimental findings,

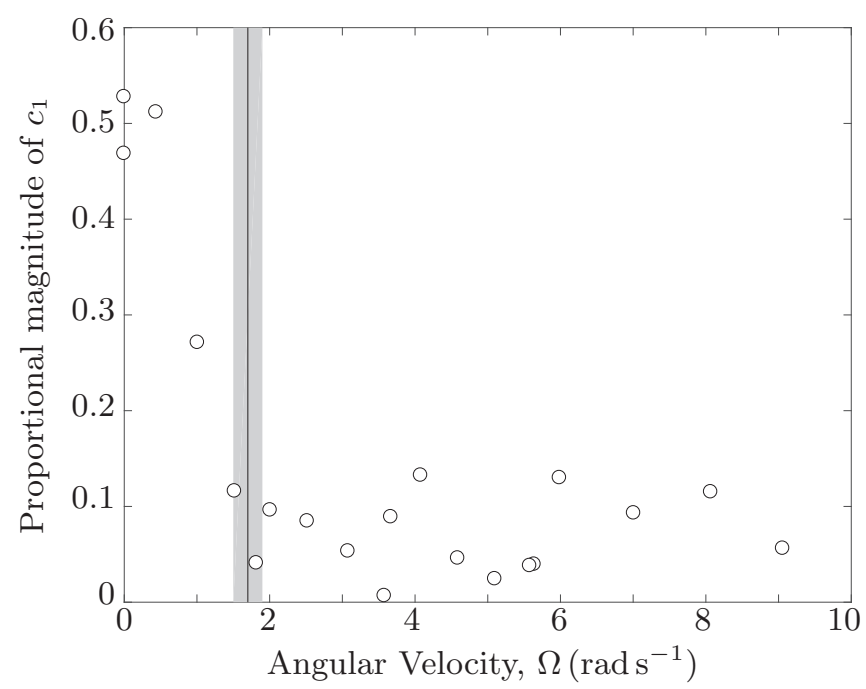

FIG. 8. The proportional mode $n=1$ contribution to the interfacial profile, when represented as a sum of Bessel functions $\mathcal{J}_{0}\left(k_{n} r / a\right)$, versus angular velocity. we conclude that the structure of the instability results from competition between: (i) rotation suppressing the larger structures, and (ii) viscosity suppressing the smaller structures. The length scale of the observed dominant mode of the instability remains an open question. We note that there is no indication of a minimum length scale in the theoretical model, which treats the liquids as inviscid.

Our experimental data show that the contribution of the axisymmetric $n=1$ mode to the instability is significantly diminished above a critical angular velocity $\Omega_{c} \approx 1.5 \mathrm{rad} \mathrm{s}^{-1}$ (Fig. 8). For comparison, we calculate the theoretical critical angular velocity required to stabilize this mode, for these experiments. For small Atwood number $0<\left|A^{\prime}\right| \ll 1$ (as in our experiments), Eq. (12) is well approximated by

$$
\Omega_{c} \approx \frac{k_{n}}{2} \frac{\sqrt{h\left|\Gamma A^{\prime}\right|}}{a},
$$

where $k_{1}=3.83$. Substituting the values of the parameters $\Gamma$, $A^{\prime}, a$, and $h$ for these experiments, given in the Appendix, we obtain $\Omega_{c}=(1.7 \pm 0.2) \mathrm{rad} \mathrm{s}^{-1}$, which agrees well with the experimental data.

We have assumed in our model that the magnetic field gradient $\partial B^{2} / \partial z$ at the interface can be treated as a constant; that is, its value at the equilibrium position of the interface at the origin. In the experiments there is some variation in this quantity over the interface, and therefore variation in the vertical component of the magnetic force on the liquids, due to the spatial variation of the magnetic field and the curvature of the equilibrium shape of the interface under rotation. We calculate that the variation in $\partial B^{2} / \partial z$ over the equilibrium surface of the interface is $\sim 10 \%$ within the window that we analyzed, $r<30 \mathrm{~mm}$, at the onset of instability. At the highest angular velocities there are some qualitative differences between the structure in the $r \lesssim 30 \mathrm{~mm}$ region and the structure closer to the edge of the tank. It is possible that these differences reflect the greater variation in $\partial B^{2} / \partial z$ in the region $r>30 \mathrm{~mm}$ : we calculate that $\partial B^{2} / \partial z$ on-axis exceeds the value at $r=50 \mathrm{~mm}$ by $25 \%$ for $\Omega=9 \mathrm{rad} \mathrm{s}^{-1}$ and $40 \%$ at $\Omega=13 \mathrm{rad} \mathrm{s}^{-1}$. In all other cases the structure of the instability is remarkably 
consistent over the whole of the interface. We also calculate that the radial gradient $\partial B^{2} / \partial r$ never exceeds $0.1 \partial B^{2} / \partial z$ at any point on the interface within the region $r<30 \mathrm{~mm}$, so that, to a good approximation, the radial magnetic force on the interface may be treated as small compared with the vertical magnetic force.

The magnetic field profile $B(r, z)$ used in our calculations was obtained from the Biot-Savart law by numerical integration in MATLAB, using the solenoid coil geometry provided by the manufacturer. The numerical calculations of the magnetic field were verified by measurement using a Gauss meter.

In these experiments the dominant magnetic forces are those that arise through the interaction of the magnetic field with the spin of the $\mathrm{Mn}^{2+}$ ions and with the molecular orbitals of the water molecules, i.e., paramagnetic and diamagnetic forces. Electromagnetically induced forces, arising from motion of the weakly conducting aqueous fluids (conductivity, $\sigma \approx 4 \mathrm{Sm}^{-1}$ ) through the magnetic field, are small in comparison. Nevertheless, they may introduce a damping effect on fluid motion that deviates from rigid body rotation [25]. Our experiments to determine the effect of viscosity on the smallest observed length scale of the dominant mode suggest that electromagnetic damping may make a significant contribution to the damping of the fluids when the viscosity is low [5]. Coriolis forces dominate electromagnetically induced forces in all the experiments under rotation: The Elsasser number $E l=\sigma B^{2} /(\rho \Omega)$, the ratio of electromagnetic to Coriolis forces, is less than $2 \times 10^{-2}$ for $\Omega \geqslant 0.5 \mathrm{rad} \mathrm{s}^{-1}$, which is the smallest nonzero rotation rate applied in our experiments. We make the assumption that the magnetic field generated by movement of the weakly conducting liquids through the magnetic field is insignificant compared to the imposed magnetic field since the magnetic Reynolds number in these experiments is very small, being of order $\operatorname{Re}_{m}=$ $\mu_{0} U L \sigma \sim 10^{-7}-10^{-8}$ for the largest length $L$ and velocity $U$ scales in our experiments [26].

In Ref. [8] we assumed that the instability develops from a state of unstable equilibrium, in which the equilibrium shape of the interface is parabolic due to the rotation. In the experimental work, the system was prepared in a state of stable hydrostatic equilibrium and then lowered into a region of magnetic field in which the direction of the effective gravity vector is inverted, with magnitude of order $|\Gamma| \sim 10 \mathrm{~ms}^{-2}$. Although the equilibrium shape in this region is an inverted parabola, the instability was observed to develop from the initial parabolic shape formed during spin-up. Hence, when the interface became unstable, its shape was not, in general, the equilibrium shape calculated for the corresponding effective gravity. That our theoretical predictions and experimental results are nevertheless consistent indicates that this difference in initial conditions between theoretical model and experiment was relatively unimportant. One reason for this may be because we have restricted our analysis to a region of the interface enclosing the rotation axis where the interface is close to horizontal so that the deviation between the observed interfacial shape and the calculated equilibrium shape is relatively minor.

We have compared the results of experiments with theory for a liquid system with an Atwood number of order $10^{-3}$.
In many situations of interest, such as in inertial confinement fusion, the Atwood number is orders of magnitude larger [2]. While we are not able to study such high Atwood number systems experimentally using the set-up described here, our theory remains applicable to these high Atwood number cases [8].

\section{CONCLUSIONS}

We have compared our theoretical model of the classical Rayleigh-Taylor instability under rotation about a vertical axis with our experimental realization of this model. Experiments showed that the length scale of the dominant mode in the instability decreased with increasing angular velocity, in agreement with our theoretical prediction that rotation tends to have a stabilizing effect on longer wavelength modes. Our observation that the overall growth rate of the instability is reduced with increasing rotation is also in agreement with the theoretical model. The measured critical angular velocity to stabilize the $n=1$ axisymmetric mode agrees well with estimates of the predicted angular velocity from theory.

A key question is whether the interface of an intrinsically unstable system $(A<0)$ could be stabilized indefinitely given a sufficiently high rotation rate. Equivalently we could ask whether it is possible to find a rotation rate rapid enough to stabilize the interface for any desired length of time. It seems reasonable to suggest that it would be possible to rotate the system quickly enough that the large-scale structures are completely suppressed by rotation, and any remaining small-scale structures are suppressed by viscosity. However, noting that the theory presented in Ref. [8] is limited to a maximum angular velocity and that the experiments presented are similarly limited, we conclude that while the instability can be suppressed, it cannot be suppressed indefinitely, at least not in the configurations considered.

\section{ACKNOWLEDGMENTS}

This work was supported by the UK Engineering and Physical Sciences Research Council (Grant No. EP/I004599/1, No. EP/K5035-4X/1); and The Leverhulme Trust (Grant No. RPG-2018-363). We thank L. Eaves and M. R. Swift for useful discussions, T. Wright and O. J. Larkin for technical support, and the referees for helpful comments and suggestions.

\section{APPENDIX}

\section{Experimental parameters}

Table I gives the parameters for the experiments on the growth rate of the instability and the experiments to measure the proportion of the mode 1 contribution to the growth.

The concentrations of the solutions $c\left(\mathrm{~mol} \mathrm{l}^{-1}\right)$ were calculated from the masses of the salts and the volume of the solutions measured during their preparation. The difference in density between the two solutions, $\Delta \rho$, and the Atwood number, $A$, were obtained using an empirical expression for the densities of binary aqueous salt solutions [27],

$$
\rho=\rho_{w}+\left(A+B \tau+C \tau^{2}\right) c+\left(D+E \tau+F \tau^{2}\right) c^{3 / 2},
$$


TABLE I. Experimental parameters.

\begin{tabular}{lc}
\hline \hline$c_{1}\left(\mathrm{MnCl}_{2}\right)$ & $(0.0614 \pm 0.0004) \mathrm{mol} \mathrm{l}^{-1}$ \\
$c_{2}(\mathrm{NaCl})$ & $(0.433 \pm 0.001) \mathrm{mol} \mathrm{l}^{-1}$ \\
$\Delta \chi=\chi_{2}-\chi_{1}$ & $(-1.14 \pm 0.01) \times 10^{-5}$ \\
$\Delta \rho=\rho_{2}-\rho_{1}$ & $(11.44 \pm 0.08) \mathrm{kg} \mathrm{m}^{-3}$ \\
$A=\Delta \rho /\left(\rho_{2}+\rho_{1}\right)$ & $(5.66 \pm 0.04) \times 10^{-3}$ \\
$\Gamma$ & $\approx-7 \mathrm{~ms}^{-2}$ \\
Tank radius $a$ & $\left(\Gamma-g=-17 \mathrm{~ms}^{-2} \pm 10 \%\right)$ \\
Layer depth $h$ & $35 \mathrm{~mm}$ \\
\hline \hline
\end{tabular}

where $\tau$ is the temperature (degree Celsius), $A-F$ are saltdependent coefficients given in [27], and $\rho_{w}(\tau)$ is the density of water. The difference in volume magnetic susceptibility between the two solutions was calculated using Curie's law, $\Delta \chi=C / T$ [28], where $C(c)$ is the Curie constant for the paramagnetic $\mathrm{Mn}^{2+}$ ions in the manganese chloride solution, and $T$ is the absolute temperature (kelvin). In this calculation, the sodium chloride solution, having no paramagnetic ions, was assumed to have the susceptibility of water. The value of $\Gamma$ given in the above table is the value of $\Gamma$ at the interface when the tank reaches the lowest point in its descent. The value of $\partial B^{2} / \partial z$ required in the calculation of $\Gamma$ [Eq. (7)] was obtained from the magnetic field profile of the solenoid coil, which was calculated from the Biot-Savart law by numerical integration.

\section{Dispersion relation in gradient magnetic field}

In this section, we calculate the dispersion relation of interfacial waves in the nonrotating two fluid system in the gradient magnetic field. We take $z=0$ to be the vertical position of the (horizontal) interface. With the addition of the magnetic body force, the Euler equation is

$$
\rho_{j} \frac{D \boldsymbol{u}_{j}}{D t}=-\nabla p_{j}+\frac{\chi_{j}}{2 \mu_{0}} \nabla B^{2}+\rho_{j} \boldsymbol{g}
$$

for the two layers $j=1,2$, where $g=-g \hat{z}$. We assume that the liquids are incompressible so that $\boldsymbol{\nabla} \cdot \boldsymbol{u}_{j}=0$. We nondimensionalize via

$$
\begin{aligned}
& \boldsymbol{x}=h_{0} \boldsymbol{x}^{*}, \quad \boldsymbol{u}=\left\{g h_{0}\right\}^{1 / 2} \boldsymbol{u}^{*}, \quad t=\left\{h_{0} / g\right\}^{1 / 2} t^{*}, \\
& B=B_{0} B^{*}, \quad p=\rho_{0} g h_{0} p^{*}, \quad \rho=\rho_{0} \rho_{j}^{*},
\end{aligned}
$$

where $\rho_{0}=\frac{1}{2}\left(\rho_{1}+\rho_{2}\right)$, which forces $\rho_{1}^{*}+\rho_{2}^{*}=2$, and $h_{0}$ is a representative length scale, e.g., $h_{0}=\min \left\{h_{1}, h_{2}\right\}$. This yields the nondimensional system

$$
\nabla^{*} \cdot \boldsymbol{u}_{j}^{*}=0, \quad \rho_{j}^{*} \frac{D \boldsymbol{u}_{j}^{*}}{D t^{*}}=-\nabla^{*} p_{j}^{*}+\chi_{j} \frac{\mathrm{Ma}}{2} \nabla^{*}\left(B^{*}\right)^{2}-\rho_{j}^{*} \hat{z},
$$

where

$$
\mathrm{Ma}=\frac{B_{0}^{2}}{\rho_{0} g h_{0} \mu_{0}}
$$

is a nondimensional number that represents the ratio of magnetic to hydrodynamic pressures. We drop the star notation immediately for clarity. The hydrostatic initial condition is given by $\boldsymbol{u}_{j}=0$ and so the corresponding hydrostatic pressure field in each layer, $p_{0 j}$, is given by

$$
\begin{gathered}
\nabla p_{0 j}=\chi_{j} \frac{\mathrm{Ma} \nabla B^{2}}{2}-\rho_{j} \hat{z}=\nabla\left[\chi_{j} \frac{\mathrm{Ma} B^{2}}{2}-\rho_{j} z\right], \\
\Rightarrow \quad p_{0 j}=f_{j}(t)+\chi_{j} \frac{\operatorname{Ma} B^{2}}{2}-\rho_{j} z
\end{gathered}
$$

for some arbitrary functions $f_{j}(t)$.

We now neglect the relatively small radial variation of $B^{2}$ compared to its vertical variation as discussed in the main text. In this case, pressure continuity in the unperturbed state requires that

$$
f_{1}(t)+\left.\chi_{1} \frac{\mathrm{Ma} B^{2}}{2}\right|_{z=0}=f_{2}(t)+\left.\chi_{2} \frac{\mathrm{Ma} B^{2}}{2}\right|_{z=0} .
$$

For magnetized fluids in general, an additional term, the magnetic normal traction $p_{n}=\mu_{0}\left(M_{2 n}^{2}-M_{1 n}^{2}\right)$, appears in the boundary condition [29], where $M_{1 n}$ and $M_{2 n}$ are the normal components of the magnetization at the interface in each of the two fluids. This term has a profound effect on the behavior of magnetized ferrofluids (see, for example, [29]). In these experiments, the magnetization of the aqueous fluids used is several orders of magnitude weaker than that of a ferrofluid exposed to the same magnetic field; here $\boldsymbol{M}=\chi \boldsymbol{H}$ with $|\chi| \sim 10^{-5}$, where $\boldsymbol{H} \approx \boldsymbol{B} / \mu_{0}$ is the magnetic field imposed by the solenoid. Hence we neglect the magnetic normal traction term, proportional to $\chi^{2}$, in comparison to the magnetic body force which is proportional to $\chi$.

Writing $B_{0}=B(z=0)$, we have

$$
f_{2}(t)=f_{1}(t)-\frac{\mathrm{Ma} B_{0}^{2}}{2}\left(\chi_{2}-\chi_{1}\right) .
$$

We introduce small perturbations to this background hydrostatic field and apply continuity of stress and the kinematic condition at the interface.

Denoting the small perturbation to the interface $\zeta=$ $\exp \{\mathrm{i}(k x+l y-\omega t)\}$, we obtain the nondimensional dispersion relation

$$
\omega^{2}=\kappa \frac{\left[\left(\rho_{2}-\rho_{1}\right)-\left(\chi_{2}-\chi_{1}\right) \frac{\operatorname{Ma}}{2} \frac{\partial B^{2}}{\partial z}\right]}{\rho_{2} \operatorname{coth}\left(\kappa h_{2}\right)+\rho_{1} \operatorname{coth}\left(\kappa h_{1}\right)},
$$

where $\kappa=\left(k^{2}+l^{2}\right)^{1 / 2}$. In terms of dimensional quantities this is

$$
\begin{aligned}
\omega^{2} & =\kappa \frac{\left[\left(\rho_{2}-\rho_{1}\right) g-\left(\chi_{2}-\chi_{1}\right) \frac{1}{2 \mu_{0}} \frac{\partial B^{2}}{\partial z}\right]}{\rho_{2} \operatorname{coth}\left(\kappa h_{2}\right)+\rho_{1} \operatorname{coth}\left(\kappa h_{1}\right)} \\
& =\Gamma \kappa \frac{\rho_{2}-\rho_{1}}{\rho_{2} \operatorname{coth}\left(\kappa h_{2}\right)+\rho_{1} \operatorname{coth}\left(\kappa h_{1}\right)},
\end{aligned}
$$

where we have defined $\Gamma$ as

$$
\Gamma=g\left[1-\frac{1}{2 g \mu_{0}}\left(\frac{\chi_{2}-\chi_{1}}{\rho_{2}-\rho_{1}}\right) \frac{\partial B^{2}}{\partial z}\right] .
$$


[1] Y. Zhou, Rayleigh-Taylor and Richtmyer-Meshkov instability induced flow, turbulence, and mixing I., Phys. Rep. 720-722, 1 (2017).

[2] Y. Zhou, Rayleigh-Taylor and Richtmyer-Meshkov instability induced flow, turbulence, and mixing II, Phys. Rep. 723-725, 1 (2017).

[3] J. J. Tao, X. T. He, W. H. Ye, and F. H. Busse, Nonlinear Rayleigh-Taylor instability of rotating inviscid fluids, Phys. Rev. E 87, 013001 (2013).

[4] G. F. Carnevale, P. Orlandi, and Y. Zhou, Rotational suppression of Rayleigh-Taylor instability, J. Fluid Mech. 457, 181 (2002).

[5] K. A. Baldwin, M. M. Scase, and R. J. A. Hill, The inhibition of the Rayleigh-Taylor instability by rotation, Sci. Rep. 5, 11706 (2015).

[6] S. B. Dalziel, Rayleigh-Taylor instability-Experiments with image analysis, Dyn. Atmos. Oceans 20, 127 (1993).

[7] G. Dimonte and M. Schneider, Turbulent Rayleigh-Taylor instability experiments with variable acceleration, Phys. Rev. E 54, 3740 (1996).

[8] M. M. Scase, K. A. Baldwin, and R. J. A. Hill, Rotating Rayleigh-Taylor instability, Phys. Rev. Fluids 2, 024801 (2017).

[9] H. Lamb, 1932 Hydrodynamics, 6th ed. (Cambridge University Press, Cambridge, 1932).

[10] M. V. Berry and A. K. GeimOf flying frogs and levitrons, Eur. J. Phys. 18, 307 (1997).

[11] N. Hirota, in Magneto-science, in Springer Series in Materials Science, edited by M. Yamaguchi and Y. Tanimoto (SpringerVerlag, Berlin, 2006), Vol. 89, p. 55.

[12] N. Hirota, T. Homma, H. Sugawara, K. Kitazawa, M. Iwasaka, S. Ueno, H. Yokoi, Y. Kakudate, S. Fujiwara, and M. Kawamura, Rise and fall of surface level of water solutions under high magnetic field, Jpn. J. Appl. Phys. 34, L991 (1995).

[13] Y. Ikezoe, N. Hirota, J. Nakagawa, and K. Kitazawa, Making water levitate, Nature 393, 749 (1998).

[14] A. T. Catherall, L. Eaves, P. J. King, and S. R. Booth, Magnetic levitation: Floating gold in cryogenic oxygen, Nature 422, 579 (2003).

[15] H. A. Pacheco-Martinez, L. Liao, R. J. A. Hill, M. R. Swift, and R. M. Bowley, Spontaneous Orbiting of Two Spheres Levitated in a Vibrated Liquid, Phys. Rev. Lett. 110, 154501 (2013).

[16] E. Beaugnon and R. Tournier, Levitation of water and organic substances in high static magnetic fields, J. Phys. III (France) $\mathbf{1}$, 1423 (1991).
[17] R. J. A. Hill and L. Eaves, Nonaxisymmetric Shapes of a Magnetically Levitated and Spinning Water Droplet, Phys. Rev. Lett. 101, 234501 (2008).

[18] C. Lorin, A. Mailfert, D. Chatain, H. Félice, and D. Beysens, Magnetogravitational potential revealed near a liquid-vapor critical point, J. Appl. Phys. 106, 033905 (2009).

[19] L. Liao and R. J. A. Hill, Shapes and Fissility of Highly Charged and Rapidly Rotating Levitated Liquid Drops, Phys. Rev. Lett. 119, 114501 (2017).

[20] In Ref. [5] we made use of an Atwood number defined in terms of an effective density of the liquids. This concept proved unhelpful in deriving the theory of the instability under rotation, since it required the inertial mass to be distinguished from the effective gravitational mass. Here the effect of the magnetic force enters only in the definition of the effective gravity, not in the Atwood number.

[21] Y. Zhou, T. T. Clark, D. S. Clark, S. G. Glendinning, M. A. Skinner, C. M. Huntington, O. A. Hurricane, A. M. Dimits, and B. A. Remington, Turbulent mixing and transition criteria of flows induced by hydrodynamic instabilities, Phys. Plasmas 26, 080901 (2019).

[22] H. T. Rossby, A study of Bénard convection with and without rotation, J. Fluid Mech. 36, 309 (1969).

[23] Y. Zhou and W. H. Cabot, Time-dependent study of anisotropy in Rayleigh-Taylor instability induced turbulent flows with a variety of density ratios, Phys. Fluids 31, 084106 (2019).

[24] S. Chandrasekhar, Hydrodynamic and Hydromagnetic Stability (Dover, New York, 1961).

[25] P. A. Davidson, An Introduction to Magnetohydrodynamics (Cambridge University Press, Cambridge, 2001).

[26] Contrast the magnetic Reynolds number in our experiments with the magnetic Reynolds number for a liquid metal with the same length and velocity scales, $\operatorname{Re}_{m} \sim 10^{-2}$, or with that of an astrophysical plasma with $\mathrm{Re}_{m} \sim 10^{10}$ or greater (due to the large length scales) in which the magnetic field lines are essentially dragged along with the flow.

[27] P. Novotný and O. Söhnel, Densities of binary aqueous solutions of 306 inorganic substances, J. Chem. Eng. Data 33, 49 (1988).

[28] C. Kittel, Introduction to Solid State Physics (John Wiley \& Sons, New York, 1996).

[29] R. E. Rosensweig, Ferrohydrodynamics (Cambridge University Press, Cambridge, UK, 1997). 